International Journal of Modern Physics C

Vol. 22, No. 12 (2011) 1419-1425

(c) World Scientific Publishing Company

DOI: $10.1142 / \mathrm{S} 0129183111017068$

\title{
AUTHOR INDEX (Volume 22)
}

Abdel-Kareem, W., Tracking of vortical structures in threedimensional decaying homogeneous isotropic turbulence

Adamatzky, A., see Martínez, G. J.

Alonso-Sanz, R., Self-organization in the battle of the sexes

Amirjanov, A., Modeling selection and extinction mechanisms of biological systems

Anastassi, Z. A., see Panopoulos, G. A.

Azuma, H. \& Ban, M., Thermal effects in Jaynes-Cummings model derived with low-temperature expansion

Ban, M., see Azuma, H.

Bantang, J. Y., see Pastor, M. G.

Bao, Z.-J., Yan, W.-J. \& Guo, C.-X., Risk assessment of attack-induced cascade in complex networks

Beck, C., see Jiang, J.

Bertoni, F., see Piccardi, C.

Bolgorian, M., Shirazi, A. H. \& Jafari, G. R., Portfolio selection using level crossing analysis

Bouferguene, A., see Krim, L. Boughaleb, Y., see Moussa, N.

Boussinot, F., Mimicking quantum mechanics using reactive programming

Bruno, O. M., see Florindo, J. B.

Cai, X.-H., see Peng, G.-H. Calatroni, L., see Piccardi, C. Cao, B.-F., see Peng, G.-H. Cao, D., see Wu, R.
12 (2011) 1373

4 (2011) 419

1 (2011) 1

7 (2011) 669

2 (2011) 133

10 (2011) 1015

10 (2011) 1015

4 (2011) 347

8 (2011) 765

1 (2011) 13

1 (2011) 35

8 (2011) 841

10 (2011) 1063

7 (2011) 661

6 (2011) 635

9 (2011) 929

9 (2011) 967

1 (2011) 35

9 (2011) 967

11 (2011) 1281
Cao, X.-B., Du, W.-B., Hu, M.-B., Rong, Z.-H., Sun, P. \& Chen, C.-L., Topology property and dynamic behavior of a growing spatial network

4 (2011) 333

Cebrat, S., see Stauffer, D.

Cesário, A. T., see Maciel, T. O.

3 (2011) 283

12 (2011) 1361

2 (2011) 169

Chakraborty, D., Jung, J.-H. \& Khanna, G., A multidomain hybrid method for head-on collision of blackholes in particle limit

Chang, W., see Lee, J.

Chang, W., see Youn, J.

Chang, Y. \& Ping, Y., Emerging cluster analysis of SCI journals and its efficiency

Chen, B., Chen, M., Zhang, Z., Xie, Y. \& Wang, B., Speed of last vehicle feedback strategy in intelligent transportation systems

Chen, B., Sun, X., Wei, H., Dong, C. \& Wang, B., Piecewise function feedback strategy in intelligent traffic systems with a speed limit bottleneck

Chen, C.-L., see Cao, X.-B.

Chen, J., see Jiang, J.

Chen, K.-Y., Tung, P.-C., Lin, S.-L. \& Tsai, M.-T., Chaos synchronization in the presence of noise

5 (2011) 517

11 (2011) 1269

11 (2011) 1227

11 (2011) 1247

7 (2011) 745

8 (2011) 849

4 (2011) 333

1 (2011) 13

12 (2011) 1409

Chen, L., Chai, Y. \& Wu, R., Modified function projective synchronization of chaotic neural networks with delays based on observer

Chen, M., see Chen, B. 
Chen, T.-L., see Zhang, G.-Q.

Chiccoli, C., Pasini, P., Evangelista, L. R., Teixeira de Souza, R. \& Zannoni, C., Lattice spin simulations of topological defects in nematic films with hybrid surface alignments

Chopard, B., see Huber, C.

Chu, S., see Fang, K.

Corso, G., see da Silva, P. C. Corso, G., see Ribeiro, R. A. Costa, A. E. B. \& de Moura, F. A. B. F., Localization of acoustic waves in onedimensional models with chaotic elasticity

Costa, K. C. O., see Ribeiro, R. A.

Crisologo, I., Monterola, C. \& Soriano, M., Statistical feature-based Craquelure classification

da Silva, L. R., see da Silva, P. C.

da Silva, P. C., Lyra, M. L., da Silva, L. R., Corso, G. \& Fulco, U. L., Critical behavior of the contact process delayed by infection and immunization periods

D'Agostino, P. P., see Iannone, $\mathrm{G}$.

Dai, S., see Wang, C.-Y.

Dai, Y., see Fang, K.

de Castro, M., see Florindo, J. B.

de Moura, F. A. B. F., see Costa, A. E. B.

de Moura, F. A. B. F., Dynamics of one-electron in a one-dimensional systems with an aperiodic hopping distribution

Deng, J., see Fang, K.

Dong, C., see Chen, B.

Dufek, J., see Huber, C.

Du, C., see Fang, K.

$\mathrm{Du}, \mathrm{H} .$, Adaptive open-plusclosed-loop control method of modified function projective synchronization in complex networks

Du, L.-G., Li, K., Kong, F.-M. \& Hu, Y., Parallel
7 (2011) 755

5 (2011) 505

10 (2011) 1093

2 (2011) 155

6 (2011) 563

2 (2011) 123

6 (2011) 573

2 (2011) 123

$11(2011) 1191$

6 (2011) 563

6 (2011) 563

5 (2011) 543

6 (2011) 581

2 (2011) 155

9 (2011) 929

6 (2011) 573

1 (2011) 63

2 (2011) 155

8 (2011) 849

10 (2011) 1093

2 (2011) 155

12 (2011) 1393
3D finite-difference timedomain method on multiGPU systems

Du, W.-B., see Cao, X.-B.

Du, W.-B., see Zhuang, Y.

El-Astal, A. H., see Sumour, M. A.

Evangelista, L. R., see Chiccoli, C.

Evangelista, T., Lavor, C. \& Rabelo, W. R. M., A new method to calculate the inconclusive coefficients in the quantum state discrimination

Ewert, F.-K., see Lüdecke, H.-J.

Famelis, I. T., Neural network-based derivation of efficient high-order RungeKutta-Nyström pairs for the integration of orbits

Fang, K., Wu, X., Du, C., Dai, Y., Chu, S., Hu, L., Deng, J. \& Feng, Y., Density functional theory investigate of the $R g F_{n}(R g=\mathrm{Kr}, \mathrm{Xe}$; $n=2,4,6)$ molecules

Feng, X. \& Kim, B. J., Location dynamics of Foreign banking in Shanghai from 1990 to 2009

Feng, Y., see Fang, K.

Florindo, J. B., de Castro, M. \& Bruno, O. M., Enhancing volumetric BouligandMinkowski fractal descriptors by using functional data analysis

Fu, B.-B., see Zheng, J.-F.

Fulco, U. L., see da Silva, P. C.

Fulco, U. L., see Ribeiro, R. A.

Gao, J. \& Wu, Z.-Y., Synchronization of complex network with non-delayed and delayed coupling via intermittent control

Gao, Z.-Y., see Wang, C.-Y.

Gao, Z.-Y., see Zheng, J.-F.

Geroyannis, V. S., Katelouzos, A. G. \& Valvi, F. N., Magnetized rotating neutron stars simulated by general-relativistic polytropic models: The numerical treatment
2 (2011) 107

4 (2011) 333

6 (2011) 649

12 (2011) 1357

5 (2011) 505

2 (2011) 95

10 (2011) 1139

12 (2011) 1309

2 (2011) 155

10 (2011) 1081

2 (2011) 155

9 (2011) 929

5 (2011) 441

6 (2011) 563

2 (2011) 123

8 (2011) 861

6 (2011) 581

5 (2011) 441

10 (2011) 1107 
Geroyannis, V. S. \& Sfaelos, I. E., Numerical treatment of rotating neutron stars simulated by generalrelativistic polytropic models: A complex-plane strategy

Gill, W., Crossing time through the fitness barrier in an asymmetric multiplicative or additive landscape in the mutation-selection model

Guarnaccia, C., see Iannone, G.

Guo, C.-X., see Bao, Z.-J.

Guo, D., see Jiang, Z.

Guo, Y., see Zhang, X.

H.Akın, Upper bound of the directional entropy of a $\mathbb{Z}^{2}$-action

Hagishima, A., see Kukida, S. Hagishima, A., see Shigaki, K. Hammoutene, D., see Krim, L. Han, Z., see Yang, T.

Hao, Q.-Y., see Sun, X.-Y.

Hoeflich, A. F., see Martínez, G. J.

Hoggan, P. E., see Krim, L.

Horikis, T. P., Analysis of optical waveguides with arbitrary index profile using an immersed interface method

Huang, H.-B., see Li, Z.-T.

Huber, C., Dufek, J. \& Chopard, B., A simple algorithm to enforce dirichlet boundary conditions in complex geometries

$\mathrm{Hu}$, L., see Fang, K.

Hu, M.-B., see Cao, X.-B.

$\mathrm{Hu}$, Y., see Du, L.-G.

Jafari, G. R., see Bolgorian, M.

Jafari, G. R., see Namaki, A.

Iannone, G., Troisi, A., Guarnaccia, C., D'Agostino, P. P. \& Quartieri, J., An urban growth model based on a cellular automata phenomenological framework

Jensen, H. J., see Peel, A.

Jia, F. \& Xie, D., Dempstershafer evidence theory-based updating rule for continuous opinions and discrete actions
Jia, T., see Zhang, X.

3 (2011) 219

Jiang, J., Metz, F., Beck, C., Lefevre, S., Chen, J., Wang, Q. A. \& Pezeril, M., Double power-law degree distribution and informational entropy in urban road networks

Jiang, X., see Tang, S.

Jiang, Z., Liang, M. \& Guo, 11 (2011) $1293 \quad$ D., Enhancing network performance by edge addition

5 (2011) 543

8 (2011) 765

11 (2011) 1211

3 (2011) 263

7 (2011) 711

3 (2011) 271

9 (2011) 1005

10 (2011) 1063

10 (2011) 1161

2 (2011) 181

4 (2011) 419

10 (2011) 1063

7 (2011) 687

7 (2011) 729

10 (2011) 1093

2 (2011) 155

4 (2011) 333

2 (2011) 107

8 (2011) 841

4 (2011) 371

5 (2011) 543

1 (2011) 85

6 (2011) 595
Jung, J.-H., see Chakraborty, D.

Kalogiratou, Z., see Monovasilis, $\mathrm{T}$.

Katelouzos, A. G., see Geroyannis, V. S.

Khanna, G., see Chakraborty, D.

Kim, B. J., see Feng, X.

Kong, F.-M., see Du, L.-G.

Korff, R., see Malarz, K.

Kosinski, R. A., see Mrowinski, M. J.

Krim, L., Bouferguene, A., Hoggan, P. E. \& Hammoutene, D., A phenomenological monte carlo simulation of a two-step dimer/monomer surface reaction

Kułakowski, K., see Malarz, K.

Kukida, S., Tanimoto, J. \& Hagishima, A., Analysis of the influence of lane changing on traffic-flow dynamics based on the cellular automaton model

Lai, D., Wu, X., Lu, H. \& Nardini, C., Learning overlapping communities in complex networks via nonnegative matrix factorization

Lan, S., Liu, Y., Liu, B., Sheng, P., Wang, T. \& Li, $\mathrm{X}$., Effect of slopes in highway on traffic flow

Lavor, C., see Evangelista, T.

Lee, J., Youn, J. \& Chang, W., Market impact and order book characteristics in the Korean futures market

Lee, J., see Youn, J.

Lefevre, S., see Jiang, J.
3 (2011) 263

1 (2011) 13

9 (2011) 883

11 (2011) 1211

5 (2011) 517

12 (2011) 1343

10 (2011) 1107

5 (2011) 517

10 (2011) 1081

2 (2011) 107

7 (2011) 719

1 (2011) 71

10 (2011) 1063

7 (2011) 719

3 (2011) 271

10 (2011) 1173

4 (2011) 319

2 (2011) 95

11 (2011) 1269

11 (2011) 1227

1 (2011) 13 
Legara, E. F., see Longjas, A. Leng, B., see Zeng, J.-B.

Li, G.-J., see Li, Z.-T.

Li, J. \& Liu, B., Semi-analytical approach to Bosonic atom-molecule conversion via feshbach resonance

Li, K., see Du, L.-G.

Li, Q., see Yang, T.

Li, X., see Lan, S.

Li, Z.-T., Li, G.-J., Huang, H.-B. \& Lu, X.-Y., Lattice Boltzmann study of electrohydrodynamic drop deformation with large density ratio

Liang, M., see Jiang, Z.

Lichtenegger, K. \& Schappacher, W., A carbon-cyclebased stochastic cellular automata climate model

Lin, M. \& Wang, G., Selforganized criticality in an earthquake model based on assortative scale-free networks

Lin, S.-L., see Chen, K.-Y.

Link, R. \& Lüdecke, H.-J., $A$ new basic one-dimensional one-layer model obtains excellent agreement with the observed earth temperature

Link, R., see Lüdecke, H.-J.

Liu, B., see Lan, S.

Liu, B., see Li, J.

Liu, C.-Q., see Peng, G.-H.

Liu, G., see Yang, T.

Liu, M.-R., see Sun, X.-Y.

Liu, Y., see Xiong, F.

Liu, Y., see Lan, S.

Liu, Z., see Tang, S.

Longjas, A., Legara, E. F. \& Monterola, C., Power law mapping in human area perception

Lopez-Ruiz, R., see PellicerLostao, C.

Lu, H., see Lai, D.

Lu, X.-Y., see Li, Z.-T.

Lucena, L. S., see Ribeiro, R. A. Lüdecke, H.-J., see Link, R.

Lüdecke, H.-J., Link, R. \& Ewert, F.-K., How natural $i$ s the recent centennial warming? An analysis of
5 (2011) 495

8 (2011) 775

7 (2011) 729

9 (2011) 919

2 (2011) 107

10 (2011) 1161

4 (2011) 319

7 (2011) 729

11 (2011) 1211

6 (2011) 607

5 (2011) 483

12 (2011) 1409

5 (2011) 449

10 (2011) 1139

4 (2011) 319

9 (2011) 919

9 (2011) 967

10 (2011) 1161

2 (2011) 181

1 (2011) 51

4 (2011) 319

9 (2011) 883

5 (2011) 495

1 (2011) 21

10 (2011) 1173

7 (2011) 729

2 (2011) 123

5 (2011) 449
2249 surface temperature records

Lyra, M. L., see da Silva, P. C.

Ma, L., see Tang, S.

Ma, Z.-Q., Xia, C.-Y., Sun, S.-W., Wang, L., Wang, H.-B. \& Wang, J., Heterogeneous link weight promotes the cooperation in spatial Prisoner's Dilemma

Macías-Díaz, J. E., A bounded finite-difference discretization of a two-dimensional diffusion equation with logistic nonlinear reaction

Maciel, T. O., Cesário, A. T. \& Vianna, R. O., Variational quantum tomography with incomplete information by means of semidefinite programs

Maghrebi, M. J., see Zarghami, A.

Malarz, K., Korff, R. \& Kułakowski, K., Norm breaking in crowd - athermal phase transition

Martínez, G. J., Adamatzky, A., Stephens, C. R. \& Hoeflich, A. F., Cellular automaton supercolliders

Mata, M.V. M., see Ribeiro, R. A.

Mazroui, M., see Moussa, N.

Meng, H., see Wang, L.

Menin, O. H. \& Rolnik, V., Quadrant-searching: A new technique to reduce the search space of the inverse problem of EIT using BEM to the direct problem

Metz, F., see Jiang, J.

Miszczak, J. A., Singular value decomposition and matrix reorderings in quantum information theory

Monovasilis, T., Kalogiratou, Z. \& Simos, T. E., Two new phase-fitted symplectic partitioned Runge-Kuttamethods

Monterola, C., see Crisologo, I. Monterola, C., see Longjas, A. Moula, B., see Samah, M.
10 (2011) 1139

6 (2011) 563

9 (2011) 883

11 (2011) 1257

9 (2011) 953

12 (2011) 1361

9 (2011) 977

7 (2011) 719

4 (2011) 419

2 (2011) 123

7 (2011) 661

3 (2011) 305

8 (2011) 825

1 (2011) 13

9 (2011) 897

12 (2011) 1343

11 (2011) 1191

5 (2011) 495

4 (2011) 359 
Moussa, N., Tarras, I., Mazroui, M. \& Boughaleb, Y., Effects of agent's repulsion in $2 D$ flocking models

Mrowinski, M. J. \& Kosinski, R. A., Cellular automata models of evacuation on staircase-like geometries

Namaki, A., Raei, R. \& Jafari, G. R., Comparing tehran stock exchange as an emerging market with a mature market by random matrix approach

Nardini, C., see Lai, D.

Nikitin, A. A., see Plyushchenkov, B. D.

Ochoa, J. G. D., Infection spread and virus release In Vitro in cell populations as a system with percolation

Ochoa, J. G. D., Stochastic time delays in regulatory networks and model reduction

Panopoulos, G. A., Anastassi, Z. A. \& Simos, T. E., A new symmetric eightstep predictor-corrector method for the numerical solution of the radial Schrödinger equation and related orbital problems

Papadopoulos, D. F. \& Simos, T. E., A new methodology for the construction of optimized Runge-KuttaNyström methods

Pasini, P., see Chiccoli, C.

Pastor, M. G. \& Bantang, J. Y., Transport efficiency of passive diffusion of viral cargos in biological cells

Peel, A. \& Jensen, H. J., Delay before synchronization and its role in latency of sensory awareness

Pei, S., see Tang, S.

Pei, W.-J., see Pu, C.-L.

Pellicer-Lostao, C. \& LopezRuiz, R., Transition from exponential to power law income distributions in a chaotic market

Peng, G.-H., Cai, X.-H., Liu, C.-Q. \& Cao, B.-F., A new lattice model of traffic flow with the consideration of

7 (2011) 661 $1(2011) 71$

4 (2011) 371

10 (2011) 1173

8 (2011) 805

3 (2011) $249 \quad$ Quartieri, J., see Iannone, G.

4 (2011) $385 \quad$ Radwan, M. A., see Sumour, 2 (2011) $133 \quad$ F. W. F., Lucena, L. S.,

6 (2011) 623

5 (2011) 505

4 (2011) 347

1 (2011) 85

9 (2011) 883

3 (2011) 297

$1(2011) 21$ the Honk effect

Penna, T. J. P., see Stauffer, D.

Pezeril, M., see Jiang, J.

Piccardi, C., Calatroni, L. \& Bertoni, F., Clustering financial time series by network community analysis

Ping, Y., see Chang, Y.

Plyushchenkov, B. D., Nikitin, A. A. \& Turchaninov, V. I., Numerical modeling of electroacoustic logging including Joule heating

$\mathrm{Pu}$, C.-L. \& Pei, W.-J., Integrating local and global routing on scale-free networks

Qin, Z., see Zeng, J.-B.

Rabelo, W. R. M., see Evangelista, $\mathrm{T}$. M. A.

Raei, R., see Namaki, A.

Rapaport, D. C., Evaluating cumulative ascent: Mountain biking meets Mandelbrot

Ribeiro, R. A., Mata, M.V. M., Costa, K. C. O., Silva, Fulco, U. L. \& Corso, G., Difficulties in the use of DFA to characterize petroleum reservoirs

9 (2011) 967

3 (2011) 283

1 (2011) 13

1 (2011) 35

11 (2011) 1247

8 (2011) 805

3 (2011) 297

8 (2011) 775

5 (2011) 543

$2(2011) 95$

12 (2011) 1357

4 (2011) 371

3 (2011) 209

2 (2011) 123

8 (2011) 825

12 (2011) 1317

4 (2011) 333

Samah, M. \& Moula, B., $A b$ Initio study of structural stability and magnetic properties of $\mathrm{Co}_{n}(n=2-10)$ : A study based on pseudopotentials DFT

4 (2011) 359

Schappacher, W., see Lichtenegger, $\mathrm{K}$.

Sekora, M. D., Extending a Hybrid Godunov method for radiation hydrodynamics to multiple dimensions

Sfaelos, I. E., see Geroyannis, V. S.

Shabat, M. M., see Sumour, M. A.
5 (2011) 457

3 (2011) 219

$6(2011) 607$

$12(2011) 1357$ 
Sheng, P., see Lan, S.

Shi, D.-M., see Zhuang, Y.

Shigaki, K., Tanimoto, J. \& Hagishima, A., A revised stochastic optimal velocity model considering the velocity gap with a preceding vehicle

Shirazi, A. H., see Bolgorian, M.

Silva, F. W. F., see Ribeiro, R. A.

Simos, T. E., see Monovasilis, T.

Simos, T. E., see Papadopoulos, D. F.

Simos, T. E., see Panopoulos, G. A.

Soriano, M., see Crisologo, I.

Sousa, A. O., see Stauffer, D.

Stauffer, D., Cebrat, S., Penna, T. J. P. \& Sousa, A. O., Love kills: Simulations in Penna ageing model

Stephens, C. R., see Martínez, G. J.

Succi, S., see Zarghami, A.

Sumour, M. A., Radwan, M. A., Shabat, M. M. \& ElAstal, A. H., Statistical physics applied to stone-age civilization

Sun, P., see Cao, X.-B.

Sun, S.-W., see Ma, Z.-Q.

Sun, X., see Chen, B.

Sun, X.-Y., Hao, Q.-Y., Liu, M.-R. \& Wang, B.-H., Flow improvement by providing partial traffic information for road users

Tang, S., Jiang, X., Pei, S., Liu, Z., Ma, L., Zhang, Z. \& Zheng, Z., Highly nonlinear complexity of interaction dynamics in scale-free networks

Tanimoto, J., see Kukida, S.

Tanimoto, J., A study of a quadruple co-evolutionary model and its reciprocity phase for various Prisoner's Dilemma game

Tanimoto, J., see Shigaki, K.

Tarras, I., see Moussa, N.

Teixeira de Souza, R., see Chiccoli, C.

Troisi, A., see Iannone, G.

Tsai, M.-T., see Chen, K.-Y.
4 (2011) 319

6 (2011) 649

9 (2011) 1005

8 (2011) 841

2 (2011) 123

12 (2011) 1343

6 (2011) 623

2 (2011) 133

11 (2011) 1191

3 (2011) 283

3 (2011) 283

4 (2011) 419

9 (2011) 977

12 (2011) 1357

4 (2011) 333

11 (2011) 1257

8 (2011) 849

2 (2011) 181

9 (2011) 883

3 (2011) 271

4 (2011) 401

9 (2011) 1005

7 (2011) 661

5 (2011) 505

5 (2011) 543

12 (2011) 1409
Tung, P.-C., see Chen, K.-Y.

Turchaninov, V. I., see Plyushchenkov, B. D.

Ubertini, S., see Zarghami, A.

Valvi, F. N., see Geroyannis, V. S.

Vianna, R. O., see Maciel, T. O.

Wang, B., see Chen, B.

Wang, B., see Chen, B.

Wang, B.-H., see Sun, X.-Y.

Wang, B.-H., see Zhuang, Y.

Wang, C.-Y., Gao, Z.-Y., Zheng, J.-F. \& Dai, S., Travel time distribution for network flows under local routing

Wang, G., see Lin, M.

Wang, H.-B., see Ma, Z.-Q.

Wang, J., see Ma, Z.-Q.

Wang, J.-F., see Wang, T.

Wang, L., see Ma, Z.-Q.

Wang, L., Zhang, H., Meng, H. \& Wang, X., The influence of individual driver characteristics on congestion formation

Wang, Q. A., see Jiang, J.

Wang, T., see Lan, S.

Wang, T. \& Wang, J.-F., An adaptive EBCA model probing the problem of riding against the traffic flow

Wang, X., see Wang, L.

Wei, H., see Chen, B.

$\mathrm{Wu}$, B. \& Romatschke, P., Shock wave collisions in $A d S_{5}$ : Approximate numerical solutions

Wu, R. \& Cao, D., Function projective synchronization of chaotic systems via nonlinear adaptive-impulsive control

Wu, R., see Chen, L.

Wu, X., see Fang, K.

$\mathrm{Wu}, \mathrm{X}$., see Lai, D.

Wu, Z.-Y., see Gao, J.

Xia, C.-Y., see Ma, Z.-Q.

Xia, X., see Yang, T.

Xie, D., see Jia, F.

Xie, Y., see Chen, B.

Xiong, F., Liu, Y. \& Zhang, Z., Dynamics with coevolution of individual inclination and opinion
6 (2011) 581

12 (2011) 1409

8 (2011) 805

9 (2011) 977

10 (2011) 1107

12 (2011) 1361

7 (2011) 745

8 (2011) 849

2 (2011) 181

6 (2011) 649

5 (2011) 483

11 (2011) 1257

11 (2011) 1257

2 (2011) 191

11 (2011) 1257

3 (2011) 305

1 (2011) 13

4 (2011) 319

2 (2011) 191

3 (2011) 305

8 (2011) 849

12 (2011) 1317

11 (2011) 1281

2 (2011) 169

2 (2011) 155

10 (2011) 1173

8 (2011) 861

11 (2011) 1257

10 (2011) 1161

6 (2011) 595

7 (2011) 745

1 (2011) 51 
Xiong, Z., see Zeng, J.-B.

Yang, L.-X., see Zheng, J.-F.

Yang, L.-X., Zhao, X.-M. \& Zheng, J.-F., Bi-directional pedestrians with a partition line

Yang, Q.-Y., see Zhang, G.-Q.

Yang, T., Li, Q., Xia, X., Zhao, E., Liu, G. \& Han, Z., How long can you enjoy blackjack with 100 chips?

Yan, W.-J., see Bao, Z.-J.

Youn, J., see Lee, J.

Youn, J., Lee, J. \& Chang, W., Stock market differences in correlation-based weighted network

$\mathrm{Yu}, \mathrm{Z}$., see Zhang, G.-Q.

Zannoni, C., see Chiccoli, C.

Zarghami, A., Maghrebi, M. J., Ubertini, S. \& Succi, S., Modeling of bifurcation phenomena in suddenly expanded flows with a new finite volume lattice Boltzmann method

Zeng, J.-B., Leng, B., Xiong, Z. \& Qin, Z., Pedestrian dynamics in a two-dimensional complex scenario using a local view floor field model
8 (2011) 775

5 (2011) 441

8 (2011) 871

7 (2011) 755

10 (2011) 1161

8 (2011) 765

11 (2011) 1269

11 (2011) 1227

7 (2011) 755

5 (2011) 505

9 (2011) 977

8 (2011) 775
Zeng, Z., see Zhang, X.

3 (2011) 263

Zhang, G., see Zhang, $\mathrm{X}$.

3 (2011) 263

Zhang, G.-Q., Yu, Z., Yang, Q.-Y. \& Chen, T.-L., $A$ simple neuron network based on Hebb's rule

7 (2011) 755

3 (2011) 305

6 (2011) 649

Zhang, H.-F., see Zhuang, Y.

Zhang, X., Zhang, G., Jia, T., Guo, Y. \& Zeng, Z., Electronic structure and optical properties of a photoluminescent tantalite: EuKNaTaO${ }_{5}$

Zhang, Z., see Chen, B.

Zhang, Z., see Tang, S.

Zhang, Z., see Xiong, F.

Zhao, E., see Yang, T.

Zhao, X.-M., see Yang, L.-X.

Zheng, J.-F., see Wang, C.-Y.

Zheng, J.-F., see Yang, L.-X.

Zheng, J.-F., Gao, Z.-Y., Yang, L.-X. \& Fu, B.-B., Synchronization behavior in discrete systems

Zheng, Z., see Tang, S.

Zhuang, Y., Shi, D.-M., Du, W.-B., Zhang, H.-F. \& Wang, B.-H., Integrating local dynamic and global static information for routing traffic on networks
3 (2011) 263

7 (2011) 745

9 (2011) 883

1 (2011) 51

10 (2011) 1161

8 (2011) 871

6 (2011) 581

8 (2011) 871

5 (2011) 441

9 (2011) 883

6 (2011) 649 